\title{
Cuatro fronteras europeas bajo la lupa. Una metodología para el análisis de los proyectos de cooperación transfronteriza (INTERREG)
}

\author{
Jaume Feliu \\ Universitat de Girona. Departamento de Geografía \\ jaume.feliu@udg.edu
}

\section{Matteo Berzi}

Universitat Autònoma de Barcelona. Departamento de Geografía matteo.berzi@uab.cat

\section{Javier Martín-Uceda \\ Roser Pastor Saberi \\ Margarita Castañer i Vivas}

Universitat de Girona. Departamento de Geografía

javier.martin@udg.edu

roser.pastor@udg.edu

mita.castaner@udg.edu

Recibido: marzo 2018

Aceptado: junio 2018

Publicado: septiembre de 2018

\section{Resumen}

El grupo de investigación APTA ha desarrollado un método de análisis para indagar sobre la cooperación transfronteriza en las diferentes fronteras europeas. Es una metodología que permite conocer detalladamente las características de los proyectos que se construyen, que da luz a la complejidad de la red de actores que se movilizan y que muestra los patrones territoriales resultantes de la cooperación transfronteriza. El texto expone cómo se ha construido la metodología, sustentada en una base de datos de proyectos INTERREG y su explotación cualitativa, cuantitativa y cartográfica, y cómo se ha aplicado para interpretar cuatro fronteras: Portugal-España, Alemania-Polonia, Austria-Italia y España-Francia. Este último caso se ha desarrollado en profundidad para mostrar las potencialidades de la metodología propuesta.

Palabras clave: cooperación transfronteriza; INTERREG; sistema de información geográfica; impactos territoriales; Francia-España 
Resum. Quatre fronteres europees sota la lupa. Una metodologia per a l'anàlisi dels projectes de cooperació transfronterera (INTERREG)

El grup d'investigació APTA ha desenvolupat un mètode d'anàlisi per indagar a l'entorn de la cooperació transfronterera en les diferents fronteres europees. És una metodologia que permet conèixer detalladament les característiques dels projectes que es construeixen, que revela la complexitat de la xarxa d'actors que es mobilitzen i que mostra els patrons territorials resultants de la cooperació transfronterera. El text exposa com s'ha construït la metodologia, sustentada en una base de dades de projectes INTERREG i la seva explotació qualitativa, quantitativa i cartogràfica, i com s'ha aplicat per interpretar quatre fronteres: Portugal-Espanya, Alemanya-Polònia, Àustria-Itàlia i Espanya-França. Aquest últim cas s'ha desenvolupat en profunditat per mostrar les potencialitats de la metodologia proposada.

Paraules clau: cooperació transfronterera; INTERREG; sistema d'informació geogràfica; impactes territorials; França-Espanya

Résumé. Quatre frontières passées à la loupe. Une méthode pour analyser les projets de coopération transfrontalière (INTERREG)

Le groupe de recherche APTA a développé une méthode d'analyse pour étudier la coopération transfrontalière entre les différentes frontières européennes. C'est une méthode qui permet de connaître de manière détaillée les caractéristiques des projets qui se mettent en place, qui révèle aussi la complexité du réseau d'acteurs mobilisés et qui montre enfin les modèles territoriaux définis par la coopération transfrontalière. Le texte présente la manière dont la méthodologie a été construite à partir d'une base de données pour interpréter quatre frontières : Portugal-Espagne, Allemagne-Pologne, Autriche-Italie, Espagne-France. Ce dernier cas d'étude est présenté de manière plus détaillée pour montrer les potentialités de la méthode proposée.

Mots-clés: coopération transfrontalière; INTERREG; système d'information géographique; impacts territoriaux; France-Espagne

\section{Abstract. Four European borders under scrutiny: A methodology for the analysis of cross-border cooperation projects (INTERREG)}

The APTA research group has developed a methodology to investigate cross-border cooperation at different European borders. The methodology allows the characteristics of the projects to be determined in detail, revealing the complexity of the network of actors that are mobilized and the territorial patterns resulting from cross-border cooperation. The text explains how the methodology has been developed using a database of INTERREG projects and their qualitative, quantitative and cartographic exploitation, and how it has been applied to interpret four borders: Portugal-Spain, Germany-Poland, Austria-Italy and Spain-France. This last case is addressed in depth to show the potential of the proposed methodology.

Keywords: cross-border cooperation; INTERREG; geographical information system; territorial impacts; France-Spain 


\section{Sumario}
1. Introducción
4. Recapitulación y futuras líneas
2. Metodología de explotación de los resultados
3. Resultados obtenidos
Referencias bibliográficas

\section{Introducción}

En los últimos años, el grupo de investigación $\mathrm{APTA}^{1}$ ha fijado su interés en el estudio de la cooperación transfronteriza y los procesos de desarrollo local en los espacios fronterizos europeos. A través de los recientes proyectos de $\mathrm{I}+\mathrm{D}^{2}$ el equipo ha profundizado tanto en el conocimiento y el análisis del área fronteriza franco-española (Feliu et al., 2013) como de otras áreas europeas para estudios comparados (Martín-Uceda y Castañer, 2018). Concretamente, se ha interesado por la incidencia territorial de los proyectos de Cooperación Territorial Europea (INTERREG), de sus lógicas, protagonistas y resultados. Ante el papel de los actores y de los proyectos de territorio, subyace la hipótesis de que el análisis de los proyectos ejecutados, de los actores involucrados, de las relaciones institucionales (estructuras de gobernanza) y socioeconómicas, pueden ser un buen indicador de procesos de desarrollo local que se gestan en dichos territorios. Frente a las experiencias de investigación acumuladas en los últimos años, se ha considerado apropiado presentar una reflexión estructurada sobre la metodología desarrollada por el grupo de investigación para analizar e interpretar los espacios transfronterizos europeos a la luz de los proyectos de cooperación entre los actores territoriales. La reflexión tiene como objetivo dar a conocer el método empleado, exponer las principales características de las cuatro fronteras europeas analizadas y demostrar su utilidad para indagar en las dinámicas de cooperación, como se muestra con la frontera España-Francia.

Desde un punto de vista analítico, los estudios de frontera, más conocidos como border studies (Jacobs and Van Assche, 2014) se han interesado por definir su delimitación espacial, su estructura interna y las dinámicas políticas (Popescu, 2008), socioeconómicas, culturales (Paasi, 1998) y territoriales (Decoville et al., 2013). La literatura académica anglófona identifica las áreas alrededor de una frontera con diferentes denominaciones como borderlands (Martinez, 2002), transfrontier regions (Anderson, 1997), cross-border regions (Perkmann, 2003) o transboundary regions, en el ámbito de las ciencias ambientales (Wolmer, 2003). Se podría definir un espacio transfronterizo como «una

1. <www.udg.edu/apta>.

2. Proyecto I+D CSO2011-26151 «25 años de proyectos transfronterizos entre España y Francia en el marco de la UE. Un análisis desde la geopolítica de planes, proyectos, agentes y resultados territoriales» (2011-2014). Proyecto I+D CSO2015-67124-R «Cooperación transfronteriza en Europa, una geopolítica de escala local. Análisis en cinco países europeos de buenas prácticas para la integración y el desarrollo global» (TRANSBORDEREUCOP). 
área más o menos extensa localizada a un lado y otro de la frontera nacional, cuyas características territoriales (geográfica, física, socioeconómica, cultural y política) están afectadas tanto por el desarrollo de las funciones de la frontera como por los cambios en las políticas nacionales, internacionales y subnacionales» (Berzi, 2017: 3). Dentro de la Unión Europea, el proceso de integración proporciona un marco legal, jurídico y unas políticas territoriales para fomentar las interacciones entre comunidades fronterizas. La cooperación transfronteriza local representa, entonces, una oportunidad para llevar a cabo la potencial puesta en marcha de procesos de desarrollo local endógeno y compartido. Sin embargo, la integración transfronteriza (Sohn, 2014) representa un proceso complejo y de larga duración, basado tanto en las relaciones horizontales (escala local) como en las interacciones verticales (multinivel), y que involucra las instituciones europeas, nacionales y regionales (véase el artículo de Berzi y Castañer en este número). Entendemos entonces la cooperación transfronteriza como «la colaboración más o menos institucionalizada que se desarrolla entre autoridades y/o entidades subestatales de uno o varios estados, y su máximo exponente es la creación de organismos de cooperación, orientada a la coordinación vertical y horizontal de políticas y acciones» (Oliveras et al., 2010).

A partir de estas premisas, el grupo APTA ha desarrollado una metodología de análisis que permite cuantificar y cartografiar todo el conjunto de proyectos transfronterizos, los actores participantes y los territorios afectados. Esta metodología ha sido diseñada para ser aplicable a cualquier frontera de la UE que se desee analizar y a partir de cualquier proyecto de cooperación transfronteriza. Hasta el momento, el método se ha aplicado para analizar los proyectos INTERREG de la frontera Francia-España, España-Portugal, Alemania-Polonia y Austria-Italia (figura 1). En este artículo se describirán los pasos metodológicos necesarios para desarrollar la metodología, para así ser reproducida por otras investigaciones. También se mostrará qué tipología de resultados se han obtenido para demostrar su utilidad en el momento de interpretar el territorio y sus dinámicas. Los resultados se estructuran en: (1) tipología de los proyectos, (2) análisis territorial del líder de proyecto, (3) análisis territorial de los actores y (4) análisis del impacto territorial del proyecto. Finalmente se propone, a la luz de los resultados obtenidos, una recapitulación y una reflexión sobre las potenciales líneas de investigación.

\section{Metodología}

Como paso previo al desarrollo de la metodología se realizó una indagación y reflexión sobre la cantidad y calidad de los datos existentes para los proyectos INTERREG europeos. Aunque la información de los proyectos no se encuentra centralizada en ninguna base común y de forma estándar, se contactó con las diferentes instituciones consignatarias de los proyectos y se comprobó la disposición de datos de calidad para el análisis esperado. Se podían obtener datos de los tres aspectos principales que afectan a los procesos de cooperación transfronteriza: la tipología de los proyectos, actores según ámbito territorial 
y financiación por institución. La tipología de los proyectos aporta la información cualitativa necesaria para clasificar y analizar temáticamente los proyectos transfronterizos. Se determinaron nueve tipos de proyectos a clasificar (figura 2). La información individualizada de los actores permite la contabilización, localización y análisis cartográfico de su actividad. Finalmente, los datos de financiación permiten un análisis cuantitativo y un valor objetivo de ponderación de las actividades de los proyectos y los actores. El resultado de la indagación previa fue positivo en determinar la capacidad de los datos y la metodología para analizar estos territorios.

La metodología se basa en dos pasos sucesivos. En primer lugar, la construcción de una base de datos que permite la introducción de diferentes campos de información de proyectos de cooperación y la cuantificación de sus diferentes aspectos. En segundo lugar, la construcción de una cartografía para interpretar conjuntamente los territorios de un lado y otro de una frontera. Los requisitos que los autores se impusieron para el diseño de la base de datos fueron:

a) permitir la introducción y clasificación de diferentes tipos de proyectos transfronterizos y de diferentes años (para poder observar su evolución);

b) ser adaptable a diferentes contextos territoriales, a diferentes fronteras europeas;

c) posibilitar la extracción de información valiosa (cualitativa y cuantitativa) sobre el número y tipología de los actores implicados en los proyectos,

d) cartografiar los resultados y operar numéricamente con los datos introducidos.

Con estos condicionantes se ha diseñado un sistema formado por tres tablas de datos relacionados con, al menos, un campo común operativo. La primera tabla (A-PROYECTOS) recoge información general sobre los proyectos transfronterizos. A cada registro se le asigna un proyecto. Los campos de información recogidos en esta tabla son los siguientes: 1) identificador del proyecto (campo común), 2) nombre del proyecto, 3) año de inicio, 4) año de finalización, 5) tipología, 6) origen de la financiación, 7) financiación total, 8) financiación europea, 9) actor jefe de fila del proyecto, 10) código geográfico del actor. La segunda tabla de datos (B-ACTORES) recoge información de todos los actores participantes en los proyectos y su función es describir y representar las características de los actores. Cada proyecto tiene varios registros, dependiendo del número de actores. Los campos estudiados son los siguientes: 1) identificador del proyecto (campo común), 2) nombre del proyecto, 3) nombre del actor, 4) tipología de actor 5) código geográfico del actor. La tercera y última tabla de datos (C-TERRITORIO) recoge los territorios donde el proyecto tiene un impacto directo, en relación con las acciones y políticas diseñadas en él. Para esta tabla, cada proyecto tiene uno o más registros, dependiendo de los territorios afectados. Los territorios no se pueden superponer y deben dibujar un área homogénea. Los campos estudiados son: 1) identificador del proyecto (campo común), 2) nombre del proyecto, 3) nombre del espacio geográfico, 4) código geográfico. 
Para la representación cartográfica de los proyectos, los actores y los territorios se ha optado por la representación areal, es decir, por el uso de capas de polígonos que se corresponden con el área propia de cada actor territorial. Así, a cada actor se le asigna un código geográfico. La gran mayoría de los códigos territoriales coinciden con los códigos oficiales europeos de las NUTS (Nomenclature of Territorial Units for Statistics): NUTS 0 (estados), NUTS 1 (grandes regiones socioeconómicas: p. ej. Länder en Alemania o Regiony en Polonia) NUTS 2 (regiones: p. ej. comunidades autónomas en España o régions en Francia, etc.) $)^{3}$, NUTS 3 (subdivisión regional: provincias en España, départements en Francia). Para los actores municipales, se utilizan los códigos oficiales europeos para esta escala administrativa, los llamados LAU (Local Administrative Units) de nivel 2, los anteriormente llamados NUTS 5.

En los ámbitos con un alcance territorial no coincidente con las clasificaciones anteriores, se han diseñado capas cartográficas y códigos geográficos ad hoc. Este es el caso de una comarca, una mancomunidad de municipios, un pays, una communauté de communes, etc. La adecuación cartográfica y de códigos también ha sido necesaria en otros casos, como en las asociaciones de regiones.

En este sentido, la gran mayoría de actores territoriales participantes en los proyectos transfronterizos se adecuan bien a las áreas geográficas mencionadas y los correspondientes códigos geográficos diseñados. Sin embargo, surgen algunas dudas en ciertos actores de más difícil adscripción territorial. Es el caso, por ejemplo, de las universidades, que pueden tener influencias territoriales bastante variadas. En este caso se ha optado por adscribirlas al ámbito correspondiente al NUT 3.

Así, la opción de dotar a cada actor de un código y de un ámbito geográfico permite representar diversos actores de diversas escalas territoriales al mismo tiempo, aunque supone unas operaciones previas de álgebra de mapas. Efectivamente, uno de los problemas que se plantean es la dificultad de representar en una misma capa de información uno o más actores que se superponen y que además no coinciden en sus límites. Por ejemplo, en el caso de representar los actores de un proyecto donde participa un LAU2 (p. ej. el municipio de Girona) y un NUT 3 (p. ej. la provincia de Girona). La solución a la problemática se resuelve mediante el álgebra de mapas, básicamente la suma de las diversas capas que representan los actores. Así, la suma del municipio de Girona (valor $=1$ ) y provincia de Girona (valor $=1$ ) tiene en cuenta los dos actores y su ámbito territorial, y refleja la doble participación del ámbito del municipio de Girona (valor $=1+1=2$ ), ya que está incluido en la provincia (figuras 3A, 4A y 5A).

Además, el álgebra de mapas permite otras operaciones interesantes para esta investigación, como la representación de los proyectos y los actores no solo en cuanto a su número absoluto, sino según una ponderación o peso. En

3. En el caso de las regiones francesas (NUT2), se han considerado las existentes en el periodo analizado, anteriores a la agregación de 2016. 
nuestro caso hemos utilizado los fondos totales destinados a cada proyecto o los fondos gestionados por cada actor para ponderar la representación cartográfica y así acercarnos más al impacto real en el territorio de los proyectos transfronterizos (figuras 3B, 4B y 5B).

La aplicación de toda esta estructura de bases de datos, como primer paso dentro del proyecto de investigación, se ha probado para representar todos los proyectos transfronterizos implicados en la financiación del POCTEFA 20072013. Y la misma estructura puede usarse para ampliar la información con los proyectos INTERREG precedentes, o incluso futuros.

Los casos de estudio que han sido, y aún son, objeto de aplicación de la metodología fueron definidos en el marco del proyecto de investigación «Cooperación transfronteriza en Europa, una geopolítica de escala local. Análisis en cinco países europeos de buenas prácticas para la integración y el desarrollo global» (TRANSBORDEURCOP). Se trata de las fronteras entre España-Francia, Portugal-España, Italia-Austria y Alemania-Polonia (figura 1). La metodología se ha aplicado completamente en toda la frontera entre los países descritos, con la excepción del caso de Alemania-Polonia, donde falta un pequeño tramo de la región de Silesia, en el sur, por dificultades en la disponibilidad de los datos a corto plazo.

Para estas fronteras se ha analizado un mismo periodo de proyectos INTERREG A, el período VI, correspondiente a los años 2007-2013. Hay que especificar que, de modo experimental, y para la frontera España-Fran-

Figura 1. Fronteras analizadas
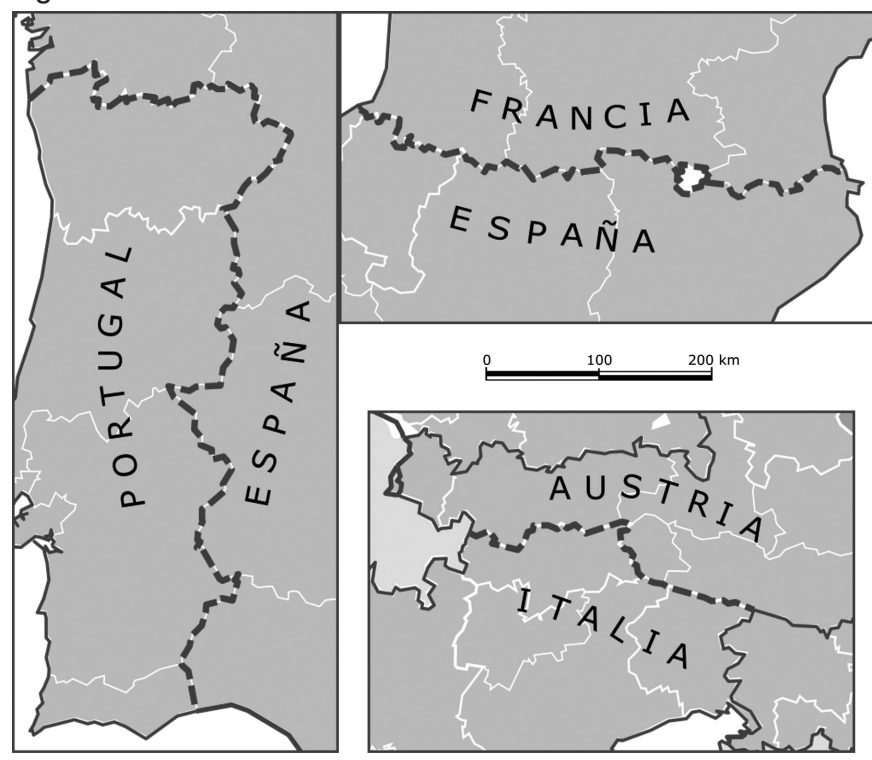

Fronteras analizadas

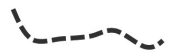

Fuente: elaboración propia. 
cia, también se ha aplicado la metodología para analizar el período anterior, INTERREG III, correspondiente a los años 2000-2006. De este modo, se ha podido realizar el análisis de una frontera durante un periodo de catorce años, entre 2000 y 2013. Los resultados fueron presentados por Berzi, M. et al. (2014). En esta investigación no se han tenido en cuenta estos datos, con la finalidad de poder comparar de forma más efectiva los cuatro casos de estudio.

\section{Resultados obtenidos}

En el marco de estas cuatro fronteras se han analizado un total de 693 proyectos transfronterizos, distribuidos tal como se muestra en la tabla 1 (fila a). Realmente, se trata de una cantidad elevada de proyectos teniendo en cuenta todo el volumen de datos que se relacionan entre sí. Para elaborar un indicador sintético de la densidad de proyectos para cada frontera, hemos relacionado el número de proyectos y la longitud de la frontera analizada (fila h). De esta manera, se observa una alta densidad de proyectos en la frontera de Alemania-Polonia $(0,46$ proyectos por $\mathrm{km})$ y una baja densidad en PortugalEspaña $(0,18)$, un hecho atribuible a factores como la densidad de población de cada frontera. Italia-Austria muestra una densidad relativamente alta $(0,37)$ y España-Francia está más cerca de los valores poco densos $(0,23)$.

Otro orden de datos recogidos es el coste total de los proyectos, así como la parte de financiación europea que han generado (fila b y c). Una primera diferencia importante entre las fronteras es el porcentaje de financiación europea, que puede suponer hasta el $83 \%$ del coste total de los proyectos en Alemania-Polonia o solo el 58\% en Italia-Austria. Esta diferencia es debida a la diversidad de las regiones elegibles para el programa INTERREG en términos de renta, donde se prioriza la financiación de países con menor renta. Por lo tanto, la mayor financiación en Alemania-Polonia también es un factor que puede explicar el mayor número de proyectos en este territorio.

Una segunda diferencia radica en la cuantía media del coste de los proyectos (fila d). A partir del indicador básico del coste total dividido por el número de proyectos de cada ámbito concluimos que todas las fronteras, a excepción de Italia-Austria, obtienen un valor parecido, unos $1.600 .000 €$ de media por proyecto. En cambio, el coste medio en Italia-Austria es tan solo de $700.000 €$ de media. Este valor tan bajo está además reforzado por el coste parecido entre todos sus proyectos (dispersión baja del 63,8\%), demostrando la clara tendencia en la frontera Italia-Austria por elaborar proyectos más reducidos de presupuesto, seguramente influidos por el menor financiamiento por parte de la Unión Europea (fila e). La dispersión del coste es elevada en Portugal-España y España-Francia, mostrando una coexistencia entre unos proyectos de presupuesto elevado y otros más modestos.

Para los diferentes casos se ha calculado un indicador simple, la longitud de la frontera (fila f), como valor para contextualizar territorialmente los datos globales de cada frontera. Se trata de un indicador menos complejo que la 
población, superficie o renta de las regiones implicadas, pero más efectivo para una primera aproximación y comparación homogénea de la distribución lineal de los valores. En este sentido, cabe destacar la frontera Alemania-Polonia en el indicador que compara el coste de los proyectos con los $\mathrm{km}$ de frontera (filla g). Muestra una densidad más elevada que los otros casos en lo que podríamos llamar «dinero invertido por km». Efectivamente, este indicador puede revelar una mayor capacidad de influencia de los proyectos en la transformación del territorio.

El número de actores implicados es otro indicador interesante en el campo de estudio de los agentes territoriales y la gobernanza (fila j). El número total de actores analizados entre los cuatro casos suma un volumen de 1.782 actores. La frontera Portugal-España, con gran diferencia respecto a los otros casos, movilizó 738 actores entre estos años (2007-2013), repartidos equitativamente entre los dos países. La frontera de España-Francia movilizó 499 actores y las restantes dos fronteras quedan a cierta distancia (298 y 247). Esta diferencia absoluta en el número de actores queda relativizada si la contextualizamos con la longitud de la frontera analizada. De esta manera, no observamos excesivas diferencias de densidad de actores en las fronteras analizadas, donde la media de los casos es de 0,67 actores por $\mathrm{km}$ de frontera (fila $\mathrm{m}$ ).

Finalmente, con relación al análisis general de los actores, se ha calculado el índice de Gini de cada frontera (fila l) para cuantificar el grado de diversificación de los actores en los diferentes proyectos, es decir, si en una frontera hay unos pocos actores que participan en numerosos proyectos o si, en cambio, la participación de los actores es equitativa. El resultado muestra una mayor concentración de actores en las fronteras de Italia-Austria y Alemania-Polonia. Por ejemplo, en el primer caso encontramos actores muy redundantes como la Autonome Provinz Bozen (22 proyectos). La frontera Portugal-España no dista mucho de estos primeros casos, y la frontera España-Francia es la que muestra una mayor distribución equitativa de actores, donde el $83 \%$ de todos los actores analizados participan exclusivamente en un solo proyecto.

Seguidamente, presentamos los diferentes resultados que profundizan en el análisis de cada frontera y que se obtienen a partir de la aplicación de la metodología descrita. Hemos dividido los resultados en distintos apartados: (1) tipología de los proyectos, (2) análisis territorial de los jefes de fila, (3) análisis territorial de los actores y (4) análisis del impacto territorial del proyecto.

En el primer apartado, describimos los cuatro casos de estudio a partir de un gráfico comparativo (figura 2) de las tipologías de los proyectos. Para el objetivo y extensión de este artículo es posible y recomendable la comparación de las cuatro fronteras. En cambio, en los apartados 2, 3 y 4 solo se muestran los mapas correspondientes a un caso de estudio, España-Francia, debido a la imposibilidad material de mostrar todos los mapas de las cuatro fronteras y los comentarios correspondientes. Ya que el objetivo del artículo es mostrar las potencialidades de la metodología de estudio, los autores han preferido ejemplificar las posibilidades cartográficas de un solo caso de estudio donde se puedan comparar los diferentes mapas extraídos. 
Tabla 1. Principales características de los casos analizados (INTERREG IV 2007-2013)

\begin{tabular}{|c|c|c|c|c|c|c|}
\hline Fila & $\begin{array}{l}\text { Frontera } \\
\text { analizada }\end{array}$ & $\begin{array}{l}\text { España- } \\
\text { Francia }\end{array}$ & $\begin{array}{l}\text { Portugal- } \\
\text { España }\end{array}$ & $\begin{array}{l}\text { Italia- } \\
\text { Austria }\end{array}$ & $\begin{array}{l}\text { Alemania- } \\
\text { Polonia }\end{array}$ & Total \\
\hline a & $\begin{array}{l}\mathrm{N} .^{\circ} \text { proyectos } \\
\text { analizados }\end{array}$ & 151 & 215 & 148 & 179 & 693 \\
\hline$b$ & $\begin{array}{l}\text { Coste total de } \\
\text { los proyectos }(€)\end{array}$ & 252.551 .272 & 343.731 .901 & 97.477 .382 & 294.189 .256 & 987.949 .811 \\
\hline c & $\begin{array}{l}\text { Financiación } \\
\text { europea }(€)\end{array}$ & 162.899 .998 & 272.583 .040 & 56.696 .500 & 243.578 .504 & 735.758 .042 \\
\hline$d$ & $\begin{array}{l}\text { Coste medio } \\
\text { del proyecto }\end{array}$ & 1.672 .525 & 1.598 .753 & 658.631 & 1.643 .515 & 1.425 .613 \\
\hline e & $\begin{array}{l}\text { Dispersión sobre } \\
\text { el coste medio } \\
\text { (C. V. de Pearson) }\end{array}$ & 174,7 & 204,1 & 63,8 & 126,7 & - \\
\hline$f$ & $\begin{array}{l}\text { Longitud de la } \\
\text { frontera analizada } \\
(\mathrm{km})\end{array}$ & 656 & 1.214 & 404 & 385 & 2.659 \\
\hline g & $\begin{array}{l}\mathrm{N} .^{\circ} \text { de proyectos/ } \\
\mathrm{km} \text { de frontera }\end{array}$ & 0,23 & 0,18 & 0,37 & 0,46 & 0,26 \\
\hline $\mathrm{h}$ & $\begin{array}{l}\text { Coste total/km } \\
\text { de frontera }\end{array}$ & 384.987 & 283.140 & 241.281 & 764.128 & 371.549 \\
\hline i & $\begin{array}{l}\text { Financiación } \\
\text { europea/km } \\
\text { de frontera }\end{array}$ & 248.323 & 224.533 & 140.338 & 632.671 & 276.705 \\
\hline j & $\begin{array}{l}\text { N. }{ }^{\circ} \text { de actores } \\
\text { implicados }\end{array}$ & 499 & 738 & 298 & 247 & 1.782 \\
\hline k & $\begin{array}{l}\mathrm{N} \cdot{ }^{\circ} \text { de } \\
\text { participaciones de } \\
\text { los actores }\end{array}$ & 636 & 1.061 & 464 & 380 & 2.541 \\
\hline I & $\begin{array}{l}\text { Índice de Gini } \\
\text { de los actores } \\
\text { implicados }\end{array}$ & 0,062 & 0,116 & 0,155 & 0,155 & - \\
\hline $\mathrm{m}$ & $\begin{array}{l}\mathrm{N} \cdot{ }^{\circ} \text { de actores } / \mathrm{km} \\
\text { de frontera }\end{array}$ & 0,76 & 0,61 & 0,74 & 0,64 & 0,67 \\
\hline
\end{tabular}

Fuente: elaboración propia a partir de la base de datos INTERREG IV (2007-2013).

\subsection{Tipología de los proyectos}

A partir del campo de la base de datos referente a la tipología de los proyectos se pueden extraer datos del conjunto de proyectos y clasificarlos según su orientación u objetivo temático. De esta manera se pueden obtener, al menos, dos informaciones relevantes. En primer lugar, un listado de tipología de proyectos según el número total de proyectos, información útil para entender por qué estrategias apuestan los diferentes territorios. En segundo lugar, cuál es la tipología de proyectos según la financiación total (o incluso la parte de financiación europea). Este dato permite detectar, por ejemplo, 
si hay proyectos que, a pesar de ser escasos en número, han requerido mucha financiación.

Si representamos esta información para los cuatro casos de estudio podemos comparar los resultados en porcentaje de proyectos por cada tipología (figura 2). La primera observación es que, en todos los casos, los proyectos de desarrollo económico local son los proyectos mayoritarios. Corresponden al $47 \%$ en la frontera Italia-Austria, 46\% en Portugal-España, 38\% en EspañaFrancia y $26 \%$ en Alemania-Polonia. Se trata de proyectos en ámbitos como la mejora de la competitividad, apoyo a pymes, fomento del turismo o de la innovación en las actividades tradicionales; es decir, proyectos que intentan mejorar las condiciones económicas de este entorno. Los proyectos que destacan en la frontera Italia-Austria están relacionados con la promoción turística (p. ej. valorización de las fortificaciones, turismo y salud), la promoción de la actividad productiva (redes rurales, producción de energía renovable) o las actividades tradicionales (recursos hídricos, producción agroalimentaria). En Portugal-España destacan los proyectos de promoción turística (itinerarios y turismo sostenible, rutas del vino) o desarrollo empresarial (nanomedicina, reciclaje, TIC). En España-Francia encontramos proyectos en innovación de actividades tradicionales (promoción de la actividad agraria, matadero transfronterizo, agricultura ecológica, gastronomía), promoción del turismo (patrimonio fortificado, turismo sostenible, patrimonio industrial), actividades productivas (energía sostenible), formación profesional (sanidad, social, turismo, TIC). En Alemania-Polonia destacan la promoción del turismo (turismo de agua y deportes acuáticos, parques naturales, albergues, geoparques), actividades productivas, (biomasa, red de pequeñas empresas), formación profesional (panadería, hostelería). En términos generales, la tipología de desarrollo económico local comprende un gran número de proyectos, pero de dimensiones más bien reducidas en términos de coste total, como se observa en el porcentaje de fondos dedicados. En todos los casos estudiados el porcentaje de financiación es menor que el porcentaje del número de proyectos.

A parte de esta tipología mayoritaria, en cada frontera destacan tipologías de proyectos según el caso. En Portugal-España y España-Francia dominan en segundo lugar los proyectos de medioambiente, que se centran en múltiples temáticas alrededor de ámbitos transversales como el agua (recursos hídricos, depuración de aguas, observatorio marino, conservación de humedales, gestión de cuencas), prevención de riesgos (incendios, sísmicos, sistemas de alerta), energías renovables, ordenación/gestión de territorios forestales, biodiversidad de aves. En estas dos fronteras son importantes también los proyectos de investigación. En España-Francia los proyectos son impulsados por centros especializados y universidades situados en las ciudades de la región para tratar temas relacionados con la biomedicina, captación de $\mathrm{CO}_{2}$, reciclaje, agricultura ecológica, alimentación, nanomateriales, etc. En Portugal-España son pocos los proyectos en número, pero de muy alta financiación, y se centran en nanotecnología, agroalimentación, automoción, medioambiente. En la frontera Alemania-Polonia destacan los proyectos de cohesión e integración social y 
Figura 2. Tipología de proyectos transfronterizos según diferentes fronteras

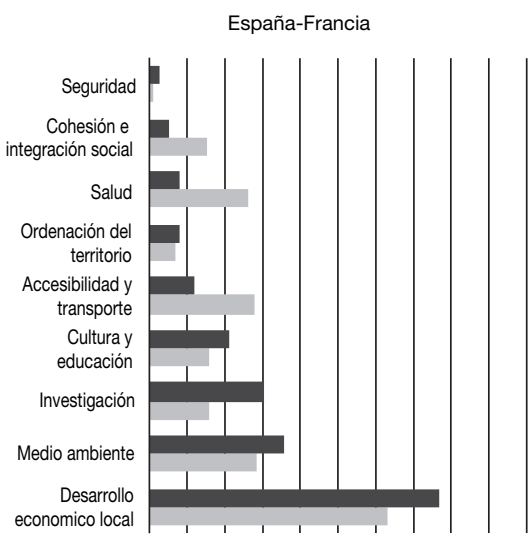

0\% $5 \%$ 10\% 15\% 20\% 25\% 30\% 35\% 40\% 45\% 50\%

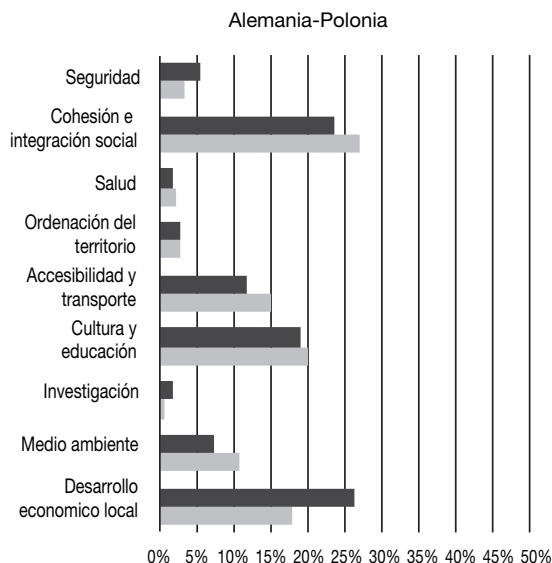

Número de proyectos
Portugal-España

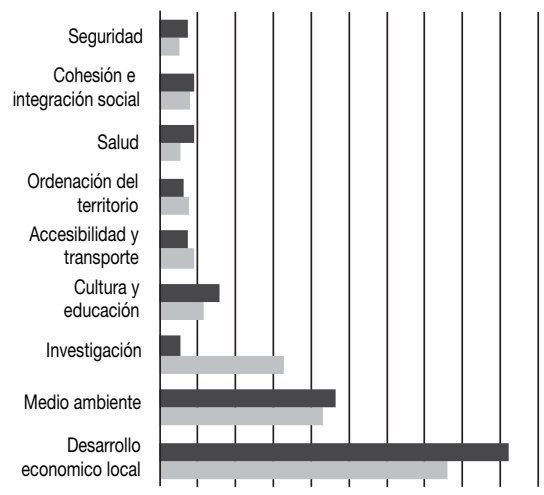

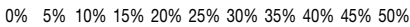

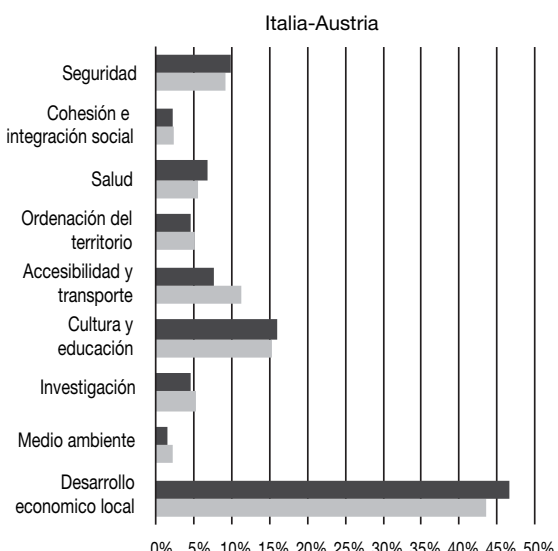

Financiación total (miles de $€$ )

Fuente: elaboración propia.

los de cultura y educación (infraestructuras de educación, deporte, museos), especialmente por el elevado coste de los mismos. Finalmente, en la frontera Italia-Austria sobresalen los proyectos de cultura y educación (red de museos, formación profesional, concienciación en temas de género, sostenibilidad, minorías lingüísticas) y los de seguridad.

\subsection{Análisis territorial de los jefes de fila}

A partir de los procedimientos cartográficos definidos anteriormente, se pueden construir mapas descriptivos de temas diversos. En primer lugar, se muestra 
la distribución y la densidad de los actores jefes de fila, es decir, los líderes del proyecto, a partir del número de los proyectos que han sido realizados. En este caso se subraya la importancia cualitativa de este actor que se responsabiliza de coordinar los demás actores, redactar el proyecto final, gestionar los recursos económicos, presentar los resultados finales, etc.

La representación cartográfica de los jefes de fila, por lo tanto, permite tener una primera aproximación del reparto de los proyectos en un territorio analizado, tal como se muestra en la figura 3A. Para el caso de España-Francia se pueden sacar varias conclusiones:

- La mayoría de los jefes de fila de los proyectos son españoles. Dentro de estos, destacan los actores localizados en la Comunidad de Aragón (30 proyectos), especialmente de Zaragoza, Navarra (21), País Vasco (19) y Cataluña (19).

- En el lado francés se observa una menor implicación en el liderazgo de los proyectos, donde destaca la región de Aquitania (19 proyectos), MediodíaPirineos (14) y Lenguadoc-Rosellón (5).

- La mayoría de jefes de fila son del ámbito regional (NUT2), y un número menor se corresponden con un actor de ámbito provincial/departamental (NUT3) o menor. Destaca la Generalitat de Cataluña (10 proyectos), que parece apostar estratégicamente por este rol, y el Gobierno de Navarra (9 proyectos), que aprovecha su ubicación geográfica de puente entre las regiones de Aquitania y País Vasco y Aragón y Mediodía-Pirineos.

- En el lado francés, sorprende el gobierno regional de Mediodía-Pirineos por no actuar de líder en ningún proyecto. Una razón de la menor participación como jefes de fila de las regiones francesas pueden ser las diferencias entre la organización territorial de los dos países, con mayor autonomía de gestión en el sur.

Por otro lado, la representación cartográfica se representa de forma ponderada por el dinero gestionado (figura 3B). De este modo, se tiene una idea más completa de qué territorios gestionan proyectos con más capacidad transformadora del territorio, es decir, que disponen de más fondos para hacerlo:

- Casi las tres cuartas partes de los recursos totales asignados son para los jefes de fila españoles y poco más de un cuarto va a las propuestas del norte, a pesar del peso relativamente importante de Aquitania.

- Cataluña y Aragón son las regiones con los jefes de fila que gestionan más recursos FEDER (24\% y 20\%, respectivamente). En cambio, sus regiones homólogas del norte, en el lado francés, son las que gestionan menos (3,9\% para Lenguadoc-Rosellón y 8\% para Mediodía-Pirineos).

- La explicación de esta disparidad, ya comentada, es la mayor facilidad de las regiones del sur de gestionar proyectos. Y debido a la colaboración homóloga (norte-sur) de tipo vertical, la concentración de un volumen importante de recursos en el sur significa la gestión de menos recursos en el norte. 
- No obstante, este desequilibrio no se produce en las regiones más occidentales, donde la repartición en la gestión por parte de los jefes de fila es más equitativa (16\% Navarra y Aquitania, 10\% País Vasco).

Figura 3. Densidad de los actores jefes de fila España-Francia (INTERREG IV 2007-2013)*
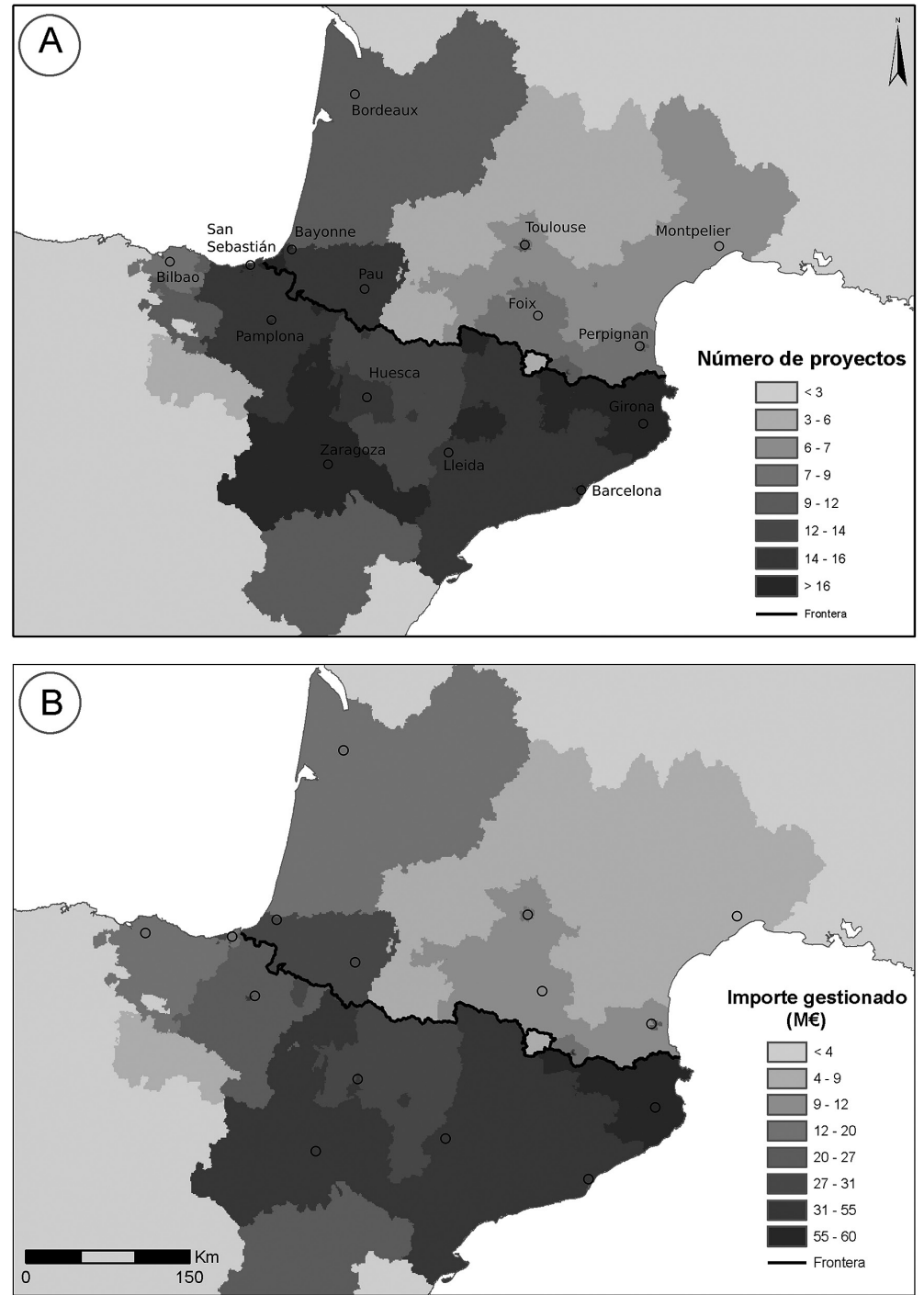

* Para facilitar la interpretación de los mapas se ha localizado la posición de las varias ciudades de referencia y se ha apuntado la toponimia de cada una de ellas solamente en el primer mapa, para permitir una lectura cartográfica más diáfana.

Fuente: elaboración propia. 


\subsection{Análisis territorial de los actores}

La representación de los actores nos aporta un punto de vista más complejo, ya que se muestra toda la diversidad de actores partícipes. El mapa sirve para indagar varios aspectos, tales como qué territorios tienen una densidad de actores más movilizados, qué equilibrios entre densidad de actores se establece a lado y lado de una frontera, qué coincidencia hay entre la movilización de actores y la red urbana de una región. Sea cual fuere el objetivo de indagación, el mapa tiene un interés especial porque consigue cartografiar los actores de un territorio con una técnica (areal) que no es habitual, ya que los actores de un territorio suelen ser cartografiados a partir de técnicas reticulares (p. ej. Chilla, T. et al, 2012).

A partir del mapa de España-Francia que representa la densidad total de actores (figura 4A) se observan características particulares del territorio:

- Mayor número de actores movilizados en la parte francesa, especialmente en el sector más occidental (municipio de Toulouse y departamento del Alto Garona; municipio de Pau y departamento de Pirineos Atlánticos). Las universidades correspondientes han tenido un rol destacado como actor.

- Mayor capacidad de movilización y participación de los actores franceses a escala de NUT3 (departamentos), a diferencia de los mismos actores a escala de las provincias españolas, que se activan más a escala de NUT2 (comunidad autónoma). En la vertiente española, no obstante, sí hay actores relevantes a nivel de NUT3 como las provincias de Zaragoza o Barcelona (universidades) o Guipúzcoa (Diputación). Pero, en general, es la escala de la comunidad autónoma la que determina los resultados en España.

- Aparte de los actores departamentales, en algunos espacios destaca la presencia de aquellos de escala territorial menor, supramunicipal, como los actores comarcales, parques naturales, les pays o las aglomeraciones francesas. Así, en la parte francesa destacan la Communauté de Communes Sud Pays Basque (un conjunto de 12 municipios franceses), Vallée d'Aspe (temática ambiental), Parc national des Pyrénées y numerosos pays (Pays Pyrénées-Méditerranée, Pays Couserans, Pays Comminges, etc.). En la parte española, en cambio, se trata mayoritariamente de entidades comarcales como Jacetania (en Aragón); la Cerdanya, Vall d'Aran, Alta Ribagorça o Pallars Sobirà (Cataluña); Bidasoa (País Vasco); Valle de Salazar y Valle del Roncal (Navarra).

- La participación de los actores a escala municipal se encuentra muy dispersa en el territorio y no coincide con la línea fronteriza. Destacan muy por encima de los otros actores municipales las grandes ciudades (especialmente San Sebastián y Toulouse, pero también Barcelona, Bilbao o Zaragoza).

- Entre otros municipios más pequeños, pero más cercanos a la frontera, destacan Bayona, Irún, Girona y Perpiñán. Finalmente, destacan el conjunto 
de municipios transfronterizos vascos, tanto en España como en Francia, por una importante concentración de actores municipales activos.

El siguiente mapa (figura 4B) se refiere a la densidad de participación de los diferentes actores ponderados por el total de los fondos que han gestionado en

Figura 4. Densidad de los actores transfronterizos España-Francia (INTERREG IV 2007-2013)
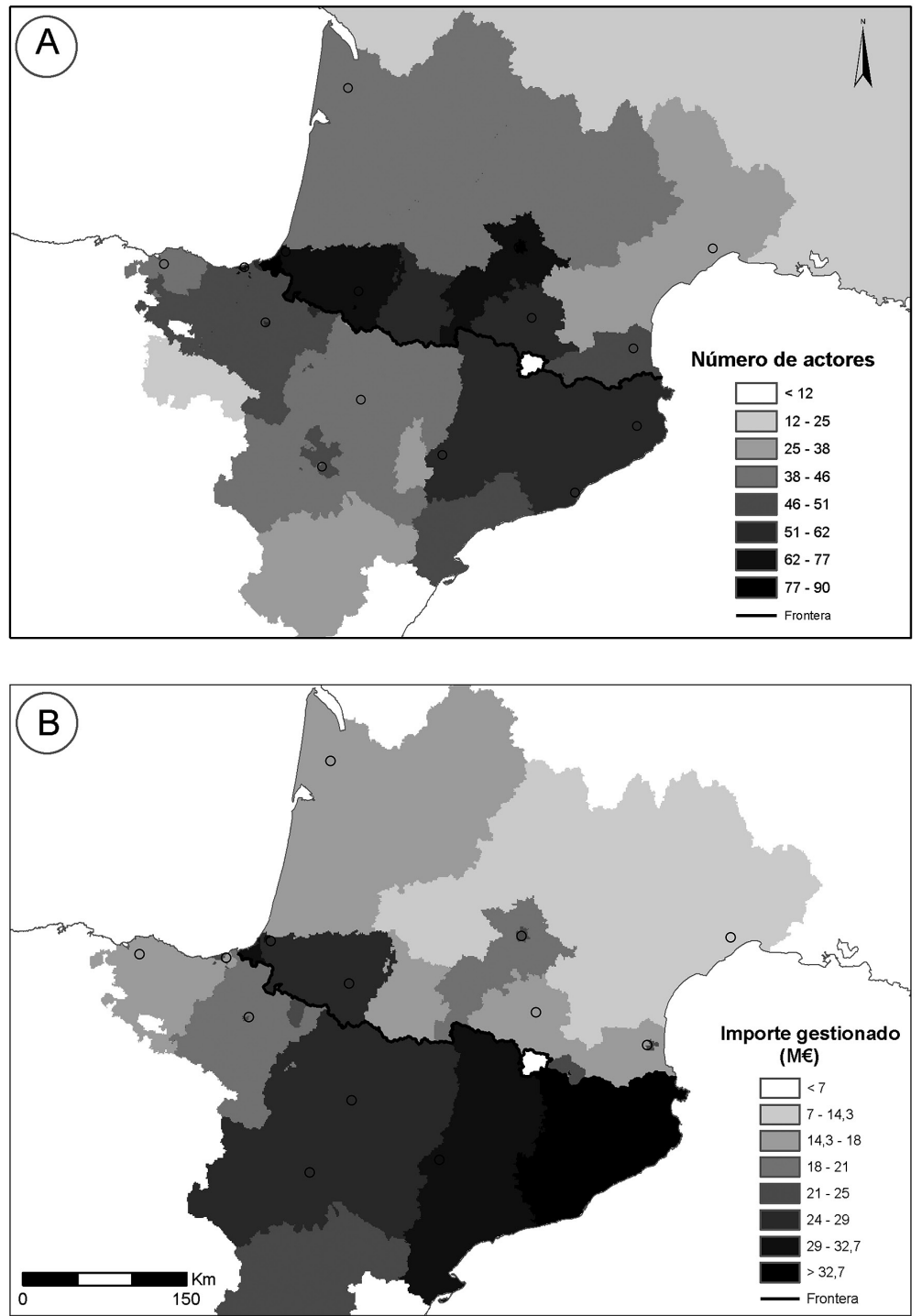

Fuente: elaboración propia. 
los proyectos. Este ejercicio nos da una mejor idea de los impactos de los proyectos de desarrollo en los diferentes territorios, si partimos de la premisa de que la mayor dotación de fondos de los proyectos por parte de los actores conlleva un mayor impacto en el desarrollo social y económico de los territorios. La imagen muestra:

- Una mayor preponderancia de los actores de la vertiente española, especialmente en el sector más oriental (provincias de Barcelona, Gerona, Zaragoza y Huesca). Los valores se explican por la significativa participación del gobierno de Cataluña en numerosos proyectos financieramente importantes, como el Hospital Transfronterizo de la Cerdanya. También existe la participación de actores de una escala menor, con una elevada aportación, como el Consell Comarcal de la Cerdanya en el proyecto del Matadero Transfronterizo, o el propio municipio de Puigcerdà en el proyecto del Hospital Transfronterizo.

- En el lado francés el panorama es prácticamente inverso al del lado español. La mayor densidad de fondos gestionados se encuentra en el Atlántico (Communauté de Communes Sud Pays Basque, municipios de Bayona y Biarritz, departamento de Pirineos Atlánticos). Las numerosas relaciones transfronterizas en el País Vasco y la función de rótula del Département des Pyrénées-Atlantiques son claves explicativas de los resultados. En el resto de los territorios franceses no aparecen densidades importantes en la cantidad de fondos gestionados (destacan el Département du Haute-Garonne y su capital Toulouse, las comarcas de la Alta Cerdanya y el Capcir y el municipio de Perpiñán).

\subsection{Análisis del impacto territorial del proyecto}

Finalmente, la base de datos permite cartografiar, gracias a la tercera tabla de datos, llamada C-TERRITORIO, los territorios concretos donde se materializan los proyectos, es decir, los espacios geográficos que son objeto de cada proyecto. Para obtener el mapa hay que interpretar, para cada proyecto, a qué espacios va dirigido. Mediante el álgebra de mapas se obtiene un mapa de la intensidad de territorios proyectuales que, probablemente, sea el más interesante desde el punto de vista del desarrollo local.

En primer lugar, se obtiene el mapa de los territorios objeto de la aplicación de los diferentes proyectos, sin ponderar con el coste del proyecto (figura 5A). Es decir, que observamos el número de veces que ciertos espacios han estado involucrados en los proyectos transfronterizos. En el caso de la frontera que ejemplificamos podemos destacar:

- En la parte francesa, hay una importante densidad de espacios de proyecto en los Pirineos Atlánticos. En este caso, se suman los 19 proyectos que tienen la Región de Aquitania como objeto, más los 35 de los Pirineos Atlánticos en su conjunto. Es decir, que esta área es objeto de múltiples proyectos que suman 
entre sí y que muestran una gran densidad. Los proyectos son variados en el ámbito de desarrollo local, medioambiente e investigación. Los otros espacios que destacan en Francia se encuentran mayoritariamente en la Región de Mediodía-Pirineos (22 proyectos), como el Alto Garona o Altos Pirineos.

- En la parte española destaca el espacio central y occidental de los Pirineos gracias a que las regiones de Aragón (25), Navarra (34) y País Vasco (24) reciben en su conjunto la atención de numerosos proyectos. Dentro de estas regiones destacan la provincia de Huesca (9) y Guipúzcoa (12).

Como último paso, si los datos sobre el número de espacios involucrados en los proyectos los ponderamos con el coste de cada proyecto (tal como hemos hecho en los mapas anteriores), surge un mapa aún más interesante desde el punto de vista del desarrollo local (figura 5B). Se visualizan los espacios donde ha recaído, mayoritariamente, el dinero procedente de los diferentes proyectos transfronterizos. Tenemos, pues, una imagen de los espacios donde se ha invertido más dinero y proyectualidad; unos espacios que son los más susceptibles de albergar procesos de desarrollo local a partir de los proyectos transfronterizos. En el caso de la frontera España-Francia destacan las siguientes características:

- Los territorios más impactados por los proyectos transfronterizos corresponden con espacios relativamente reducidos, colindantes con la frontera y distribuidos de forma alternada a lo largo de toda la frontera entre España y Francia.

- Estas características vienen a demostrar la eficiencia de la entidad gestora de las ayudas INTERREG en esta frontera (en este caso la Comunidad de Trabajo de los Pirineos) en distribuir el impacto territorial de los proyectos de forma eficiente (concentrada) y equitativa (distribuida) a lo largo de la frontera.

- En el tramo más oriental de la frontera destacan espacios como la Cerdanya española y francesa, y las comarcas colindantes. El territorio de la Cerdanya es objeto de proyectos con un alto presupuesto como el Hospital Transfronterizo de la Cerdanya (28,6 M€) o el Matadero Transfronterizo de la Cerdanya (3,6 M€).

- En la parte central correspondiente con Aragón, destaca la zona al norte de Bielsa por la gestión de numerosos proyectos a escala regional, pero especialmente por el proyecto de mejora del túnel de Bielsa-Aragnouet a través de nuevas medidas de seguridad (16,5 M€). También destaca el territorio de proyecto correspondiente a la reapertura de la línea ferroviaria internacional Pau-Canfranc (3,6 M€).

- En el tramo occidental correspondiente a Navarra y el País Vasco destacan, en primer lugar, los espacios de la llamada Eurociudad vasca, con un número elevado de proyectos de dimensión media en ámbitos variados como el desarrollo local (centro transfronterizo para el sector de energías renovables y eficiencia energética, 4,2 M€), medioambiente (conservación de espacios naturales transfronterizos: Marismas de Txingudi y Corniche Basque, 2,8 M€) o cultural (Centro Coreográfico Transfronterizo, 1,5 M€). En el resto del 
área se encuentran proyectos infraestructurales (creación de un itinerario de circulación no motorizada de Bayona a Pamplona, $8 \mathrm{M€}$ ), de desarrollo local (promoción de la actividad agraria en Navarra, Aquitania y País Vasco, 5,2 M€; valorización turística y cultural del patrimonio fortificado de Pamplona y Bayona, 4,8 M€) o ambiental (Bosque de Bosques en Irati, 3,9 M€).

Figura 5. Impacto territorial de los proyectos España-Francia (INTERREG IV 2007-2013)
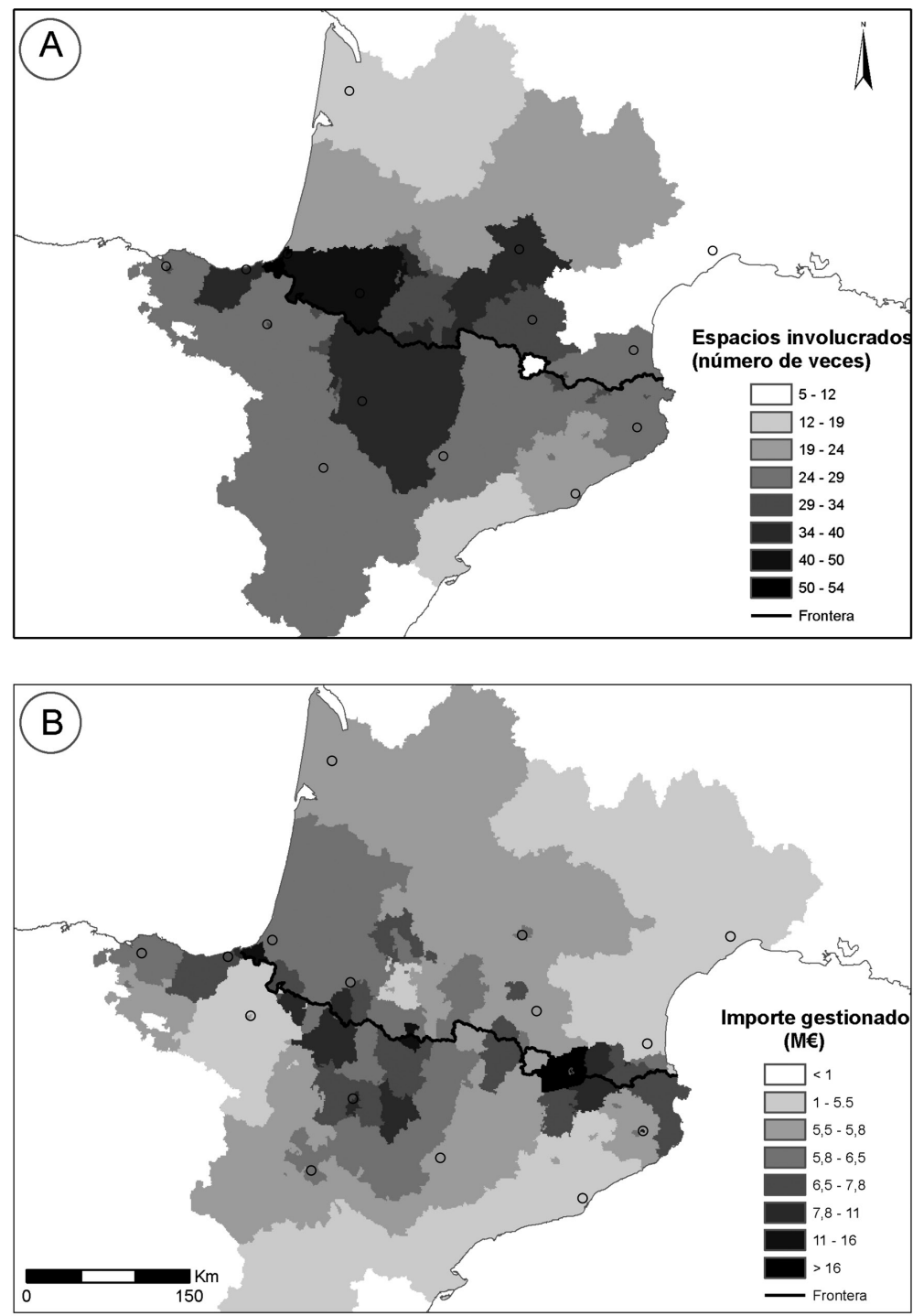

Fuente: elaboración propia. 


\section{Recapitulación y futuras líneas de explotación de los resultados}

Después de lo expuesto hasta ahora podemos defender la utilidad de una base de datos como la presentada para analizar las múltiples características de la cooperación transfronteriza, especialmente aquellas referentes a la tipología de los proyectos, su liderazgo, los actores participantes y los territorios implicados en ella. En última instancia, el análisis de la cooperación transfronteriza y sus protagonistas debe facilitar la interpretación de los procesos de desarrollo local que se producen en unos territorios específicos, transfronterizos.

La construcción y explotación de la base de datos se fundamenta en una metodología que ha sido descrita para ser comprendida y reproducida por otros grupos de investigación y territorios. Parte de unas premisas flexibles que permiten el trato de gran variedad de proyectos transfronterizos, de diferentes años, con diferentes contextos territoriales, con información cualitativa y cuantitativa y, finalmente, con capacidad de análisis cartográfico de los resultados. Las variables que se analizan y los resultados que se observan pueden utilizarse para comprobar y avanzar en las teorías sobre cooperación transfronteriza y dinámica de los territorios fronterizos presentados en el apartado teórico.

El método puede considerarse exitoso en tanto que se ha aplicado sin problemas para todo el período INTERREG IV (2007-2013) y para las fronteras entre España-Francia, Portugal-España, Italia-Austria y AlemaniaPolonia; es decir, en una variedad representativa de fronteras europeas. Para el conjunto de las cuatro fronteras se han analizado un total de 693 proyectos transfronterizos a lo largo de $2.659 \mathrm{~km}$ de fronteras, que han gestionado un volumen total de cerca 1.000 millones de euros, 736 millones de los cuales procedentes de los fondos FEDER (el 74\%), con un coste medio por proyecto de 1,4 millones de euros. También se ha analizado un volumen total de 1.782 actores territoriales participantes en los proyectos, de varias escalas y naturalezas administrativas. Todo este volumen de datos supone una información de gran valor para estudiar casos concretos de fronteras, la comparación entre ellas, o la posible ampliación hacia nuevos territorios que sumen valor a los datos.

En este artículo se han presentado los principales potenciales de la explotación de estos datos a partir de la lectura conjunta de todos los resultados y de la ejemplificación a partir de un solo caso, por razones de espacio y de oportunidad de profundización: la frontera España-Francia. En el ámbito de la tipología de los proyectos, se observan características como la preeminencia en todas las fronteras de los proyectos de desarrollo económico local (mejora de la competitividad, apoyo a pymes, fomento del turismo o de la innovación en las actividades tradicionales) y la diversidad en las otras tipologías según la frontera tratada: medioambiente en Portugal-España y España-Francia, cohesión social en Alemania-Polonia, cultura y educación en Italia-Austria. En el ámbito del análisis territorial de los actores y el impacto territorial, a la luz de los seis mapas expuestos, se han observado las siguientes características: 
- Predominancia de los actores-líder en el lado español, que además suelen ser de ámbito regional (NUT2), por razones seguramente de autonomía y capacidad administrativa.

- Lógica longitudinal en la estructuración de proyectos, que implica unas alianzas y unos equilibrios entre actores norte-sur, más que este-oeste.

- Mayor movilización general de actores NUT3 en Francia (departamentos) y mayor movilización de NUT2 en España (comunidades autónomas), que muestra cómo la diferencia en la organización territorial de los estados tiene sus consecuencias en la cooperación territorial transfronteriza.

- En este sentido, a escala local, relevante movilización de agrupaciones supramunicipales, de distinta naturaleza según el estado y, especialmente en España, según la comunidad autónoma. Así, se activan actores como las Communauté d'Agglomération y las Communauté de Communes en Francia, las comarcas en Cataluña y Aragón, y distintas formas de mancomunidades en Navarra y el País Vasco. La movilización a escala municipal también es numerosa, especialmente en los municipios mayores y, por lo tanto, con mayor capacidad económica y administrativa.

- Gran diversidad en el resto de actores (universidades, cámaras de comercio, parques naturales), que acaban conformando una red compleja de actores que interactúan de forma muy equilibrada, tal como muestran los indicadores del índice de Gini.

- Finalmente, distribución equitativa y concentrada de los territorios más densos en proyectualidad a lo largo de la frontera. Estos se convierten en territorios susceptibles de albergar procesos de desarrollo local, inducidos, en última instancia, por los fondos europeos.

Aparte de los resultados expuestos hasta el momento, los autores creen en las enormes potencialidades de la base de datos construida para explorar otras facetas de los territorios transfronterizos. En primer lugar, la base de datos permite una explotación parcial de resultados según el interés temático de la investigación. Por ejemplo, permite la selección, y el trabajo singularizado, de proyectos de una sola tipología. De esta manera, puede realizarse una investigación sobre los proyectos de cultura y educación, con datos relacionados de número de proyectos, presupuestos, características de los actores implicados, territorios impactados, etc. Otro ejemplo sería una investigación sobre una tipología de actores y su movilización en el territorio, como el rol de las universidades en los proyectos transfronterizos, qué temática de trabajo, qué alianzas con otros actores, cuánto presupuesto gestionado.

En segundo lugar, la base de datos permite una explotación relacional de los actores implicados en los proyectos, es decir, la visualización de las redes de relación que se establece entre los actores, a partir de los proyectos transfronterizos que comparten. En este caso, la base de datos puede utilizarse para representaciones reticulares en vez de las representaciones areales (álgebra de polígonos) que han sido presentadas aquí. Este análisis relacional tendría, al menos, dos formatos según si los nodos están georreferenciados o no. Podría representarse 
la red de agentes que surge de los actores participantes en los proyectos en forma de diagrama de relaciones o de diagrama de relaciones georreferenciado a partir de software especializado como Tulip u otros, que ha sido ampliamente utilizado por varios investigadores para representar la red de actores de un territorio.

En tercer lugar, la estructuración de los datos permite otras posibilidades cartográficas de las descritas hasta ahora, como la representación singularizada de los niveles administrativos participantes. Es decir, que pueden cartografiarse por separado los niveles NUT1, NUT2, NUT3, LAU2, etc., según los intereses de la investigación en curso. Por ejemplo, se podría representar solamente las agrupaciones de municipios que participan en los proyectos si fuera interesante para un estudio de esta escala administrativa y su movilización en redes de cooperación transfronteriza. Finalmente, en el ámbito cartográfico, puede ser interesante la representación en tres dimensiones de los mapas reproducidos anteriormente. Los mapas publicados muestran la densidad de un valor (ya sea número de actores, valor de los proyectos, etc.) y esta densidad está representada por una gama de color, en este caso de grises. Pero en algunos mapas (p. ej. la figura 5B), la densidad elevada se concentra en un espacio muy reducido y puede dificultar su apreciación. Una representación en 3D resolvería esta dificultad y permitiría imágenes más sugerentes de los fenómenos transfronterizos en proceso.

\section{Referencias bibliográficas}

ANDERSON, M. (1997). «Transfrontier co-operation-history and theory». En: G. BRUNN and P. SCHMITT-EGNER (eds.). Grenzüberschreitende Zusammenarbeit in Europa: Theorie - Empirie - Praxis. Baden-Baden: Nomos, 78-97.

BERZI, M. (2017). «The cross-border reterritorialization concept revisited: the territorialist approach applied to the case of Cerdanya on the French-Spanish border». European Planning Studies, 0 (0), 1-22. $<$ https://doi.org/10.1080/09654313.2017.1321622>

Berzi, M.; Feliu, J.; Vicente, J. y Castañer, M. (2014). «The cross border cooperation along Pyrenees after 2000: projects, stakeholders and territorial impacts between Spain and France». Communication at Association for Borderland Studies 1st World Conference 9-13 June 2014, Joensuu, Finland - St. Petersburg, Rusia.

Chilla, T.; Evrard, E. y Schulz, C. (2012). «On the territoriality of cross-border cooperation: "Institutional Mapping" in a multi-level context». European Planning Studies, 20 (6), 961-980.

Decoville, A.; Durand, F.; Sohn, C. y Walther, O. (2013). «Comparing Cross-border Metropolitan Integration in Europe: Towards a Functional Typology». Journal of Borderlands Studies, 28 (2), 221-237. <https://doi.org/10.1080/08865655.2013.854654>

Feliu, J.; Berzi, M.; Vicente, J.; CASTAÑ̃er, M. y Llussà, R. (2013). «Análisis de los proyectos y actores transfronterizos España-Francia en el período 2007-2013». Geographicalia, 63-64, 75-93.

JACOBS, J. y VAN AsSCHE, K. (2014). «Understanding empirical boundaries: a systems-theoretical avenue in border studies». Geopolitics, 19 (1), 182-205. $<$ https://doi.org/10.1080/14650045.2013.830106> 
Martín-UCeda, J. y CASTAÑER, M. (2018). «Actores y proyectos transfronterizos en las fronteras ibéricas: análisis comparativo a partir del IV INTERREG». Boletín de la Asociación del $A G E$.

MartineZ, O. J. (2002). "The dynamics of border interactions. new approaches to border analysis». En: C. H. SCHOfIELD (ed.). Global boundaries. World boundaries, 1, 1-15. Nueva York: Routledge.

Oliveras, X.; DurÀ, A. y Perkmann, M. (2010). "Las regiones transfronterizas: Balance de la regionalización de la cooperación transfronteriza en Europa (19582007)». Documents d'Analisi Geogràfica, 56 (1), 21-40.

PAASI, A. (1998). "Boundaries as social processes: territoriality in the world of flows». Geopolitics, 3 (1), 69-88. <https://doi.org/10.1080/14650049808407608>

Perkmann, M. (2003). "Cross-border regions in Europe». European Urban and Regional Studies, 10 (2), 153-171. <https://doi.org/10.1177/0969776403010002004>

POPESCU, G. (2008). «The conflicting logics of cross-border reterritorialization: Geopolitics of Euroregions in Eastern Europe». Political Geography, 27 (4), 418-438. <https://doi.org/10.1016/j.polgeo.2008.03.002>

SoHn, C. (2014). «Modelling cross-border integration: the role of borders as a resource». Geopolitics, 19 (3), 587-608. <https://doi.org/10.1080/14650045.2014.913029>

Wolmer, W. (2003). «Transboundary conservation: the politics of ecological integrity in the great limpopo transfrontier park». Journal of Southern African Studies, 29 (1), 261-278.

<https://doi.org/10.1080/0305707032000060449> 